\title{
ON THE EXTENSION OF MODULAR MAXIMAL IDEALS ${ }^{1}$
}

\author{
BERTRAM YOOD
}

1. Introduction. Let $B$ be a complex commutative Banach algebra and let $A$ be a subalgebra of $B$. In $[1, \S 23]$ and [3] Silov, about twenty years ago, investigated conditions under which some or all of the modular maximal ideals of $A$ are contained in modular maximal ideals of $B$. We re-examine this question for noncommutative Banach algebras. Here neither $A$ nor $B$ need be commutative and the ideals in question are the modular maximal two-sided ideals ${ }^{2}$ of $A$ and $B$. Simple examples show that, even if $A$ is a commutative subalgebra of $B$ and Silov's conditions are fulfilled, no m.m. ideal of $A$ need be contained in a m.m. ideal of $B$. Success can be hoped for only if $A$ is favorably situated in $B$.

Suppose (for simplicity) that $A$ is closed in $B$. Consider the set $\mathfrak{Q}$ of all m.m. ideals $N$ of $B$ such that $x y-y x \in N$ for all $x \in A, y \in B$. Each $N \in \mathfrak{Q}$ determines a multiplicative linear functional $x \rightarrow x(N)$ on $A$. Let $B$ be the set of m.m. ideals of $A$ which are the null spaces of multiplicative linear functionals on $A$. The algebra $A$ can be represented homomorphically as an algebra of continuous functions on $B$ in the fashion of Gelfand. Let $\Delta(A)$ be the Silov boundary of $A$ in 3. Then ${ }^{3}$ each $M \in \Delta(A)$ is contained in a m.m. ideal of $B$ if $\sup |x(N)|=\nu(x)$ for all $x \in A$ where the sup is taken over $\mathfrak{Q}$ and $\nu(x)$ is the spectral radius of $x$. A sufficient condition for this relation to hold on $A$ is that $x y-y x$ lie in the radical $J$ of $B$ for all $x \in A$, $y \in B$. An example shows that this can take place where $A$ properly contains $C+J$ where $C$ is the center of $B$.

2. Algebraic preliminaries. Let $B$ be a ring with a subring $A$ and denote by $\mathfrak{M}(\mathfrak{R})$ the set of all m.m. ideals of $A(B)$. Let $I$ be a twosided ideal of $B$ and set $C(I)=\{x \in B \mid x y-y x \in I$ for all $y \in B\}$. It is readily seen that $C(I)$ is a subring of $B$. We assume that $C(I) \supset A$. Given $M \in \mathfrak{M}$ we let $\alpha(M ; I)$ denote the set of all finite sums of the

Received by the editors May 3, 1962.

1 This research was supported by the National Science Foundation Grant NSF. G-14111.

${ }^{2}$ We shall consider exclusively two-sided ideals and refer to modular maximal two-sided ideals as m.m. ideals.

${ }^{3}$ Consider the special case where $B$ (and so $A$ ) is commutative. Here $\mathfrak{Q}$ is the set of all m.m. ideals of $B$ so that automatically sup $|x(N)|=\nu(x)$ for all $x$, the sup taken over $\mathfrak{Q}$. As $\mathfrak{Z}$ is here the set of all m.m. ideals of $A$, our theorem, in this case, reduces to Silov's theorem [1, p. 213]. 
form $\sum x_{k} z_{k}+u$ where each $x_{k} \in M, z_{k} \in B$ and $u \in I$. We set $\beta(M ; I)$ $=\{w \in B \mid w y \in \alpha(M ; I)$ for all $y \in A\}$. Since $C(I) \supset A$ it is clear that $\alpha(M ; I)$ can be described as the set of all sums $\sum z_{k} x_{k}+u$ so that $\alpha(M ; I)$ is a two-sided ideal of $B$. Also, $\beta(M ; I)=\{w \in B \mid y w \in \alpha(M ; I)$ for all $y \in A\}$ so that $\beta(M ; I)$ is a two-sided ideal of $B$ containing $M$ and $I$.

We let $J$ denote the radical of $B$ and $S$ its strong radical (the intersection of its m.m. ideals), see [2, p. 59]. Note that $S \supset J$.

2.1. Lemma. If $\beta(M ; I) \neq B$ there exists $N \in \Re$ such that $N \cap A=M$. If $I$ is the strong radical $S$ of $B$ and there exists $N \in \mathfrak{N}$ such that $N \cap A$ $=M$ then $\beta(M ; S) \neq B$.

Suppose that $\beta(M ; I) \neq B$ and let $j$ be an identity for $A$ modulo $M$. Take $z \in B$ and $y \in A$. We can write $z y=y z+v$ where $v \in I$. Then $(j z-z) y=(j y-y) z+j v-v \in \alpha(M ; I)$. Therefore $j z-z \in \beta(M ; I)$ and likewise $z j-z$ for all $z \in B$. Thus $\beta(M ; I)$ is a proper modular twosided ideal of $B$ containing $M$ and is therefore contained in some $N \in \mathfrak{R}$. Since $j \notin N$ we see that also $N \cap A=M$.

Next take the case $I=S$. Suppose $N \in \mathfrak{N}$ and $N \cap A=M$. Since $N \supset S$ we also see that $N \supset \alpha(M ; S)$. If $\beta(M ; S)=B$ then $j^{2} \in \alpha(M ; S)$ $\subset N$. Since $j^{2}-j \in M \subset N$ it follows that $j \in N \cap A=M$ which is impossible.

The following example shows that, even for Banach algebras $C(J)$ can be larger than $C+J$ where $C$ is the center of $B$. Let $B$ be the Banach space of all complex-valued continuous functions on $[0,1]$ made into a Banach algebra by defining products by the rule $f g(t)$ $=f(0) g(t), 0 \leqq t \leqq 1$. For this algebra, $J=S=\{f \in B \mid f(0)=0\}, C=(0)$ and $C(J)=B$.

3. Extension of maximal ideals. We adopt the notation of $\$ 2 \mathrm{ex}$ cept that $A$ and $B$ are complex Banach algebras (with $A$ algebraically embedded in $B$ ). Let $\mathfrak{Q}=\{N \in \mathfrak{N} \mid x y-y x \in N$ for all $x \in A, y \in B\}$. For each $N \in \mathfrak{N}$ let $x \rightarrow \pi_{N}(x)$ be the natural homomorphism of $B$ onto $B / N$. The latter is a simple Banach algebra with an identity. If $N \in \mathfrak{Q}$, the image of $A$ in $B / N$ lies in the center of $B / N$. But that center is a field and so, by the Gelfand-Mazur theorem, is the set of scalar multiples of its identity $\pi_{N}(v)$ (see $[2$, p. 85]). If we write $\pi_{N}(x)=x(N) \pi_{N}(v)$ where $x(N)$ is a scalar, the mapping $x \rightarrow x(N)$ is a multiplicative linear functional on $A$ (trivial if $N \supset A$ ). Set

$$
\beta(x)=\sup _{N \in \mathfrak{Q}}|x(N)|, \quad x \in A .
$$

Let $\mathbb{B}$ denote the subset of $\mathfrak{M}$ consisting of all zero sets of multi- 
plicative linear functionals on $A$. For each $M \in Z$ denote the corresponding functional by $x(M)$. Using the Gelfand theory we can represent $A$ homomorphically as an algebra of continuous functions vanishing at infinity on 8 where we give to $B$ its weak topology defined by the functions $x(M), x \in A$. We may then speak of the Silov boundary $\Delta(A)$ of $A$ in $B$ in the usual way [2, p. 132]. Set

$$
\alpha(x)=\sup _{M \in 3}|x(M)|, \quad x \in A .
$$

It is clear that

$$
\beta(x) \leqq \alpha(x), \quad x \in A .
$$

For each $x \in A$ let $\|x\|_{0}(\|x\|)$ be its norm as an element of the Banach algebra $A(B)$. Consider the spectral radii $\nu_{A}(x)=\lim \left\|x^{n}\right\|_{0}^{1 / n}$ and $\nu_{B}(x)=\lim \left\|x^{n}\right\|^{1 / n}$ of $x$ computed for $A$ and $B$ respectively. The relation $\nu_{B}(x) \leqq \nu_{A}(x)$ is automatic. If $A$ is a closed subalgebra of $B$, $\nu_{B}(x)=\nu_{A}(x)$ for $x \in A$. We shall also have occasion to consider the spectrum of $x$ computed in $A(B)$ which we denote by $\operatorname{sp}(x \mid A)$ $(\operatorname{sp}(x \mid B))$.

In the notation above, for $x \in A$ we have, for each positive integer $m$,

$$
|x(N)|\left\|\pi_{N}(v)\right\|^{1 / m}=\left\|\pi_{N}\left(x^{m}\right)\right\|^{1 / m} \leqq\left\|x^{m}\right\|^{1 / m} .
$$

Letting $m \rightarrow \infty$ we see that

$$
\beta(x) \leqq \nu_{B}(x), \quad \alpha(x) \leqq \nu_{A}(x), \quad x \in A .
$$

Let $E(\mathfrak{Q})$ denote the set of $M \in \mathfrak{M}$ for which there exists $N \in \mathfrak{Q}$ such that $N \cap A=M$.

3.1. TheOREM. The following statements are equivalent.

(a) $\nu_{A}(x)=\nu_{B}(x)=\beta(x)$ for all $x \in A$.

(b) $E(\mathfrak{Q}) \supset \Delta(A)$ and $\alpha(x)=\nu_{A}(x), x \in A$.

Suppose (a) holds. It follows from (1) and (2) that $\alpha(x)=\beta(x)$, $x \in A$. Let $k(\mathfrak{Q})$ denote the intersection of the $N \in \mathfrak{Q}$ and let $M_{0} \in \Delta(A)$. By Lemma 2.1 it is sufficient to show that $\beta\left(M_{0} ; k(\mathfrak{Q})\right)$ $\neq B$ inasmuch" as any $N \in \mathfrak{R}$ such that $N \supset \beta\left(M_{0} ; k(\mathfrak{Q})\right)$ contains $k(\mathfrak{Q})$ and so lies in $\mathfrak{Q}$. Suppose the contrary. We can then write (where $j$ is an identity for $A$ modulo $M_{0}$ )

$$
j^{2}=\sum_{k=1}^{n} x_{k} z_{k}+u
$$

where each $x_{k} \in M_{0}, z_{k} \in B$ and $u \in k(\mathfrak{Q})$. 
For each $N \in \mathfrak{Q}$ consider the identity $\pi_{N}(v)$ of $B / N$. In the quotient algebra norm, $\left\|\pi_{N}(v)\right\| \geqq 1$. An equivalent norm $\left\|\pi_{N}(z)\right\|_{1}$ may be introduced into $B / N$ so that $\left\|\pi_{N}(v)\right\|_{1}=1$. We suppose that this procedure has been followed for each $N \in \mathfrak{Q}$ and set

$$
\|w \mid\|=\sup _{N \in \mathfrak{Q}}\left\|\pi_{N}(w)\right\|_{1}, \quad w \in B .
$$

Note that $\||w|\|$ is defined on all of $B$ whereas $\alpha(x)$ and $\beta(x)$ are only defined on $A$. It is easy to see that

$$
\||| x||=\beta(x), \quad x \in A .
$$

Our argument is now an adaptation of one of Silov $[1, \S 23]$. Without loss of generality we may assume that, in (3), $\beta\left(x_{k}\right) \leqq 1$ for $k=1, \cdots, n$. Let $a$ be any positive number, $a>\max \|\| z_{k} \mid \|$ and $2 n a>1$. Note that $j\left(M_{0}\right)=1$ and $x_{k}\left(M_{0}\right)=0, k=1, \cdots, n$. Consider the neighborhood $\mathfrak{B}$ of $M_{0}$ in $B$ defined by the inequalities

$$
\begin{aligned}
& \left\{M \in \Re|| x_{k}(M) \mid<1 /(2 n a), k=1, \cdots, n\right\} \\
& \left\{M \in \Re|| j^{2}(M)-1 \mid<1 / 3\right\} .
\end{aligned}
$$

From $[2$, p. 135] there exists $y \in A$ such that

$$
\sup _{M \in \mathfrak{B}}|y(M)|=1, \quad \sup _{M \notin \mathfrak{B}}|y(M)|<1 /(2 n a) .
$$

Now, for $M \in \mathfrak{B}$, we have $\left|j^{2} y(M)\right|=\left|j^{2}(M)\right||y(M)| \geqq 2|y(M) / 3|$ by (6) so that

$$
\beta\left(j^{2} y\right) \geqq 2 / 3 \text {. }
$$

Since $z_{k} y-y z_{k} \in k(\mathfrak{Q})$ we can from (3) write

$$
j^{2} y=\sum_{k=1}^{n}\left(x_{k} y\right) z_{k}+w
$$

where $w \in k(\mathfrak{Q})$. Note that $\pi_{N}(w)=0$ for each $N \in \mathfrak{Q}$. Therefore we get from (4), (5) and (9) that

$$
\beta\left(j^{2} y\right) \leqq \sum_{k=1}^{n}\left\|x _ { k } y \left|\left\|\left|\| z_{k}\right|||\right.\right.\right.
$$

Next, for $M \in \mathfrak{B},\left|x_{k} y(M)\right| \leqq\left|x_{k}(M)\right|<1 /(2 n a)$ while for $M \notin \mathfrak{B}$, $\left|x_{k} y(M)\right| \leqq \alpha\left(x_{k}\right)|y(M)| \leqq|y(M)|<1 /(2 n a)$ so that, by (5), $\left\|x_{k} y \mid\right\|$ $\leqq 1 /(2 n a)$. From (10) we see that $\beta\left(j^{2} y\right) \leqq 1 / 2$. This is contrary to (8) and we have shown that (a) implies (b).

Suppose (b) and let $x \in A$. By the definition of the Silov boundary 
there exists $M_{0} \in \Delta(A)$ such that $\left|x\left(M_{0}\right)\right|=\nu_{A}(x)$. Let $j$ be an identity for $A$ modulo $M_{0}$ and let $N \in \mathfrak{Q}$ have the property that $N \cap A=M_{0}$. As $j^{2}-j \in M_{0} \subset N$ and $j(N) \neq 0$ we see that $j(N)=1$. Suppose $x\left(M_{0}\right)$ $=a$. Then $x-a j \in N$ whence $|x(N)|=\nu_{A}(x)$. Therefore $\beta(x) \geqq \nu_{A}(x)$ and, by (2), we see that $\nu_{A}(x)=\nu_{B}(x)=\beta(x)$.

3.2. Lemma. If $C(J) \supset A$ then $\mathfrak{Q}=\mathfrak{N}$ and $\beta(x)=\nu_{B}(x)$ for all $x \in A$.

Since $J \subset S$ it follows from $C(J) \supset A$ that $\mathfrak{Q}=\mathfrak{R}$. Let $x \in A$ and let $a \in \mathrm{sp}(x \mid B), a \neq 0$. Set $K=\left\{a^{-1} x y-y \mid y \in B\right\}+J$. Clearly $K=\left\{a^{-1} y x-y \mid y \in B\right\}+J$ and is a modular two-sided ideal of $B$. Suppose that $K=B$. Then there exists $y \in B, z \in J$ such that $a^{-1} x+y$ $-a^{-1} x y=z$. This implies that $a^{-1} x$ is right quasi-regular in $B$. Likewise $a^{-1} x$ is left quasi-regular and hence quasi-regular in $B$ which is impossible. Thus there exists $N \in \mathfrak{R}$ with $K \subset N$. Since $J \subset N$ it follows that $\pi_{N}\left(a^{-1} x\right)$ is the identity of $B / N$. Therefore $x(N)=a$. Conversely, if $x(N)=a \neq 0$ for some $N \in \mathfrak{N}$ then $\left\{a^{-1} x y-y \mid y \in B\right\}$ lies in $N$ so that $a \in \operatorname{sp}(x \mid B)$. Thus $\beta(x)=\nu_{B}(x)$.

Lemma 3.2 gives a sufficient condition for the applicability of Theorem 3.1. It follows immediately that if $A$ is a closed subalgebra of the center $C$, each $M \in \Delta(A)$ is contained in some $N \in \Re$.

We next consider the strong structure space $\mathfrak{M}(\mathfrak{N})$ of $A(B)$ in its hull-kernel topology [2, p. 78]. For the notion of a completely regular Banach algebra see $[2$, p. 83$]$. We can be more specific than in Theorem 3.1 if $A$ is completely regular.

3.3. Theorem. Suppose that $A$ is completely regular and that $\nu_{A}(x)=\nu_{B}(x)=\beta(x)$ for all $x \in A$. Then $E(\mathfrak{Q})=3$.

Let an identity be adjoined to $B$ forming $B_{1}$ and let $A_{1}$ denote the corresponding augmentation of $A$. Let $\mathfrak{M}_{1}\left(\mathfrak{N}_{1}\right)$ be the strong structure space of $A_{1}\left(B_{1}\right)$ and let $\mathfrak{Q}_{1}=\left\{N_{1} \in \mathfrak{N}_{1} \mid w v-v w \in N_{1}\right.$ for all $w \in A_{1}$, $\left.v \in B_{1}\right\}$. If we write $w=\lambda+x, v=\mu+y$ where $\lambda, \mu$ are scalars, $x \in A$ and $y \in B$ we see that $N_{1} \in \mathfrak{V}_{1}$ if and only if $x y-y x \in N_{1}$ for all $x \in A$, $y \in B$. The mapping $N_{1} \rightarrow N_{1} \cap B$ takes $\mathfrak{N}_{1} \sim\{B\}$ onto $\mathfrak{N}$. We then have $N_{1} \in \mathfrak{Q}_{1}, N_{1} \neq B$ if and only if $N_{1} \cap B \in \mathfrak{Q}$ and all elements of $\mathfrak{Q}$ are obtainable in this way. Next we note that $\mathfrak{N}_{1}$ is compact $[2$, p. 79] and that $\mathfrak{Q}_{1}$ is a closed subset of $\mathfrak{N}_{1}$. The mapping $\sigma: N_{1} \rightarrow N_{1} \cap A_{1}$ is a mapping of the compact set $\mathfrak{Q}_{1}$ into $\mathfrak{M}_{1}$ which is continuous (see $\left[2\right.$, p. 85]). Also $\mathfrak{M}_{1}$ is a Hausdorff space $\left[2\right.$, p. 84] so that $\sigma\left(\mathfrak{Q}_{1}\right)$ is closed in $\mathfrak{M}_{1}$.

The mapping $\tau: M_{1} \rightarrow M_{1} \cap A$ is a homeomorphism of $\mathfrak{M}_{1} \sim\{A\}$ onto $\mathfrak{M}$. But $\sigma\left(\mathfrak{Q}_{1}\right) \sim\{A\}$ is closed in $\mathfrak{M}_{1}$ so that $\tau\left[\sigma\left(\mathfrak{Q}_{1}\right) \sim\{A\}\right]$ is closed in $\mathfrak{M}$. This set is the same as $E(\mathfrak{Q})=\{N \cap A \mid N \in \mathfrak{Q}, N D A\}$. 
Recall that, by Theorem $3.1, E(\mathfrak{Q}) \supset \Delta(A)$. From our definitions $\Delta(A)$ is dense in 3 so that also $E(\mathfrak{Q}) \supset \mathfrak{Z}$. Since the reverse inequality is clear, the proof is complete.

\section{REFERENCES}

1. I. M. Gel'fand, D. A. Raikov and G. E. Silov, Commutative normed rings, Uspehi Mat. Nauk 1 (1946), 48-146; Amer. Math. Soc. Transl. (2) 5 (1957), 115-220.

2. C. E. Rickart, General theory of Banach algebras, Van Nostrand, New York, 1960.

3. G. E. Silov, On the extension of maximal ideals, Dokl. Akad. Nauk SSSR 29 (1940), 83-84.

UNIVERSITY OF OREGON

\section{SEMI-HOMOGENEOUS FUNCTIONS}

LOUIS V. QUINTAS AND FRED SUPNICK

1. Introduction and statement of results. A function $f$ is called homogeneous of degree $n$ with respect to the set $A$, or briefly semihomogeneous if

$$
f(a x)=a^{n} f(x)
$$

is satisfied for all $x$ in the domain of $f$ and all $a$ in $A$.

With each admissible $A$ there is associated a class of solutions of (1.0). E.g., let $R$ denote the set of all real numbers and let $f$ be a function on $R$ to $R$. If $A$ consists only of the irrationals, then $f(x)$ $=c x^{n}(c=f(1))$ is the unique solution of (1.0). On the other hand, if $A$ consists only of the rationals, then in addition to $f(x)=c x^{n},(1.0)$ has other solutions (e.g., if $n$ is any nonzero integer and $f(x)=x^{n}$ or 0 accordingly as $x$ is rational or irrational).

We are interested in studying decompositions of the set of admissible $A$ 's into classes and in characterizing the solutions of (1.0) corresponding to these classes. In this paper we show how this can be done in a natural way for semi-homogeneous functions of a real variable. We note that in this case our methods apply to

$$
f(a x)=p(a) f(x) \quad(a \in A \subset R),
$$

where $p$ is a product-preserving function on $R$ to $R$ (cf. [1]). We shall therefore confine our attention to (1.1).

Received by the editors March 8, 1962. 